\title{
AN ADAPTIVE MULTI-CHANNEL SPECTROELLIPSOMETER FOR ECOLOGICAL MONITORING
}

\author{
F. A. Mkrtchyan, V.I. Kovalev, V.V. Klimov \\ V.A. Kotelnikov's Institute of Radioengineering and Electronics, RAS \\ Vvedensky Sq.,1, Fryazino, Moscow region,141190 Russia, Email:ferd47@mail.ru
}

KEY WORDS: Spectroellipsometry, GIMS-Technology, Multi-channel Spectroellipsometer, Expert System, Pollution spots, Classification, Identification.

\begin{abstract}
:
The creation of multichannel polarization optical instrumentation and use of spectroellipsometric technology are very important for the real-time ecological control of aquatic environment. Spectroellipsometric devices give us high precision of measurements.

This report is aimed to describe:

-A technology of combined use of spectroellipsometry and algorithms of identification and recognition that allowed the creation of a standard integral complex of instrumental, algorithmic, modular and software tools for the collection and processing of data on the aquatic environment quality with forecasting and decision - making functions.

-A compact measuring - information multichannel spectroellipsometric system (device) for monitoring the quality of aquatic environment, that is based on the combined use of spectroellipsometry and training, classification, and identification algorithms.

This spectroellipsometric system will differ from modern foreign analogues by the use of a new and very promising method of ellipsometric measurements, an original element base of polarization optics and a complex mathematical approach to estimating the quality of a water object subjected to anthropogenic influence.

Unlike foreign analogues, the system has no rotating polarization elements. This allows one to increase the signal-to-noise ratio and the long-term stability of measurements, to simplify and reduce the price of multichannel spectroellipsometers.

The system will be trainable to the recognition of the pollutants of aquatic environment.

A spectroellipsometer in laboratories of V.A. Kotelnikov's Institute of Radioengineering and Electronics, Russian Academy of Sciences is designed for in-situ real time measurements of spectra of ellipsometric parameters Psi and Delta with consequent changeover to spectra of transmitted and reflected signal from water media in frames of used physical model of water environment.
\end{abstract}

\section{GIMS- TECHNOLOGTY}

Water ecosystems are one of the important objects of geoinfomational monitoring. Knowledge of microwave attenuation properties of water systems needed in this respect since attenuation values and their dependence on frequency and biometrical features afford a basis for microwave remote sensing retrieving algorithms.

One of perspective approach to the solution of the problems arising here is GIMS-technology(GIMS = GIS + model). The basic scheme of collection and processing of the information in geoinformation monitoring system(GIMS) recognizes that effective monitoring researched object is possible at complex use of methods of simulation modeling, collection and processing of the information.

From the position of system analysis, the system of collection and processing of the information in geoinformation monitoring represents the structure uniting the computers of various classes, databases and the advanced problem-oriented software. Creation of such system demands the development of formalized description of the information flows and unique methodology of its processing.

Development of GIMS requires the decision of a set of problems related to the formation of data measurements flows to be solved. The problem of monitoring water ecosystems using the remote sensing measurements is one of important among them. Various algorithms of the theory of images recognition, statistical decisions and cluster analysis are used to solve this problem.

The feature of remote measurements is information acquisition, when the data of measurements, acquired during tracing of flying system along routes of survey, are directed to input of the processing system. As result the two dimensional image of investigated object is registered. Statistical model of spottiness for investigated space is one of models for this image.

In real conditions, the study of spots, the acquiring of their statistical characteristics and their using in a problem of detection is enough a complex problem. It is necessary to develop the criteria allowing the distinguishing the spots from other phenomena. For example, it is necessary to determine such threshold the exceeding of which is the spot indicator. Also it is necessary to develop model presentation of processes of spots detection.Realization of GIMS-technology will result in the creation of regional and global maps of water systems radiative characteristics and characteristics of electromagnetic waves attenuation by water system based on created database, a priori information and modeling in conditions of limited closure of Earth surface by satellite microwave survey.

\section{ABOUT METHOD OF CLASSIFICATION}

At present time there are many methods of recognition which are caused appreciably by variety of statements of concrete tasks. The problem of recognition consists in the division of some group of objects into the classes at the base of certain 
requirements. The objects having general properties are related to one class. An initial data for the solution of a recognition problem are results of some observations or the direct measurements, that are named initial attributes.

Method of taxonomy (clustering) is one of the important methods of recognition and classification of images.

Let's assume set of $\mathrm{M}$ it is required to divide by not crossed subsets (clusters), and the elements included in the same clusters should be close to each other enough from the point of view of the chosen criterion of nearness, and elements from. different clusters should be far enough from each other. In one of many possible statements of this task two numbers $a$ and $b(0$ $<a<b$ ) are given. It is considered, that two elements $\mathrm{x}$ and $\mathrm{y}$ are close to each other enough, if $p(x, y)<a$, and are far enough from each other, if $p(x, y)>b$.

Kings method is one of well-known methods of taxonomy, which gives good results in those cases, when the quantity of the available information in assumed clusters is moderate. According to this method the distance between groups of points in space of attributes is defined as distance between centers of masses of these groups. Clustering in this case is based on the assumption that sites of the increased density in space of attributes correspond to similar situations.

\section{MULTI-CHANNEL SPECTROELLIPSOMETER(MSE)}

The creation of multichannel polarization optical instrumentation and use of spectroellipsometric technology are very important for the real-time ecological control of aquatic environment. It should be mentioned that efficient solution of this multiparametric problem greatly depends on the precision and simplicity of ellipsometric devices.

This report is aimed to describe:

-a technology of combined use of spectroellipsometry and algorithms of identification and recognition that allowed the creation of a standard integral complex of instrumental, algorithmic,

modular and software tools for the collection and processing of data on the aquatic environment quality with forecasting and decision - making functions

-a compact measuring - information MSP for monitoring the quality of aquatic environment, that is based on the combined use of spectroellipsometry and training, classification, and identification algorithms(Figure 1).

This MSE will differ from modern foreign analogues by the use of a new and very promising method of ellipsometric measurements, an original element base of polarization optics and a complex mathematical approach to estimating the quality of a water object subjected to anthropogenic influence.

Unlike foreign analogues, the system has no rotating polarization elements. This allows one to increase the signal-tonoise ratio and the long-term stability of measurements, to simplify and reduce the price of MSE.

The system will be trainable to the recognition of the pollutants of aquatic environment.

- New approach in ellipsometry, based on binary polarization modulation;

- New low cost effective polarization elements;

- No rotating polarization elements;

- Excellent signal-to-noise ratio and the long-term stability of measurements makes possible to simplify and reduce the price of the MSE;

- One of the key element of the systems is a polarization switch which transforms unpolarized light from a source into highly linearly polarized light with alternate (up to $\mathrm{KHz}$ or more) and orthogonal polarizations;

- Sets of silicon photodiodes with arbitrary access to them;

- Flexibility, simple design, low cost, high precision, long term stability.

\section{Specifications MSE:}

a)Specification of portable 128-channel spectropolarimeter :

- Spectral range 280-600 nm;

- Minimal measurement time $0.5 \mathrm{sec}$;

Precision and stability 0.01 and 0.02 degree in Psi and Delta, respectively, polarization rotation angle $-0,001$ degree:

- Sources: miniature pulsed xenon lamp PX-2 with high resource and laser diode;

Micro spot focus $300 \mu \mathrm{m}$ with PX-2 and $30 \mu \mathrm{m}$ with laser diode

- Acromatic compensator ;

- User-friendly software;

- A weight of measuring device - about $4 \mathrm{~kg}$.

b)Specification of compact 128-channel spectroellipsometer with halogen lamp:

- Spectral ranges-380-740 $\mathrm{nm}$ and 650-930 nm.

- Minimal measurement time- $0.6 \mathrm{sec}$.;

- Precision to 0.003 and 0.01 degree in Psi and Delta, respectively, polarization rotation angle $-0,001$ degree. Sources: halogen lamp KGM-9-70;

- Long-term stability 0,01 degree;

- Use of acromatic compensator on the basis of Fresnel rhomb made of fused quartz enhances precision of measurements;

- A weight of measuring device - about $4 \mathrm{~kg}$;

- Polarization block-2 kg. Analyzer block-2 kg .

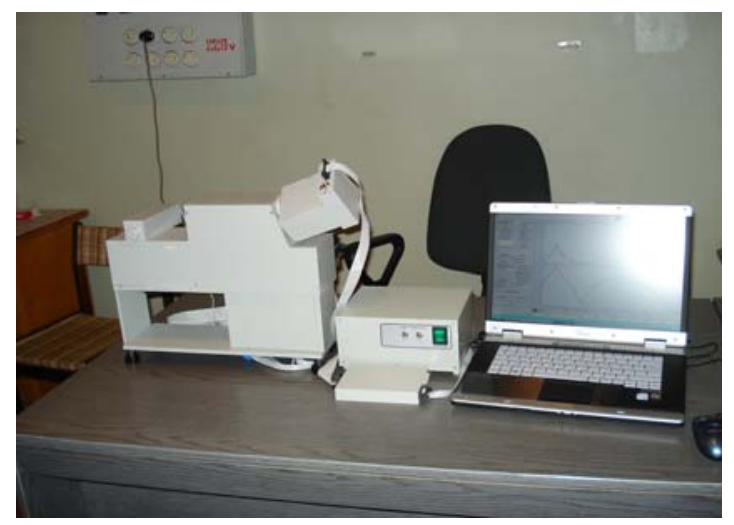

Figure 1. High precision real-time Multi-channel spectrellipsometer.

Device composition:

- Polarizer block;

- Analyzer block;

- Power supply unit;

- Illuminator;

- Fiber-optic cabel;

- Notebook with interface unit;

- Wide-band filters.

The algorithmic support of the MSP is based on a complex application of recognition and classification algorithms on the basis of 128 spectra images registered during a fixed period of time (Mkrtchyan et al., 2009; Ferdenant A. Mkrtchyan et al., 2009). 
A time interval of 1 second is usually established and provides about 30 value of brightness for each of the 128 optical channels. The spectra obtained are sources of set of statistical parameters and different characteristics united into vector spaces for their comparison with the standard samples of famous pollutants stored on the computer.

The technology of this comparison depends on the diversity of identification methods.

The system is trainable to the recognition of the pollutants of aquatic environment.

\section{MODEL OF SPOTTINNES}

The feature of remote measurements is information acquisition, when the data of measurements, acquired during tracing of flying system along routes of survey, are directed to input of the processing system. As result the twodimensional image of investigated object is registered. Statistical model of spottiness for investigated space is one of models for this image. In real conditions, the study of spots, the acquiring of their statistical characteristics and their using in a problem of detection is enough a complex problem. It is necessary to develop the criteria allowing the distinguishing the spots from other phenomena. For example, it is necessary to determine such threshold the exceeding of which is the spot indicator. Also it is necessary to develop model presentation of processes of spots detection. The method of the thresholds determination in the most obvious and simple way for spots definition. In this case that part of space belongs to area of spots, on which the parameter of environment measured within the chosen channel exceeds value $\left(l^{+}\right)$or, on the contrary, does not exceed value $\left(l^{-}\right)$ a threshold. Let

$y=y\left(x_{1}, x_{2}\right)$ is function of coordinates $\left(x_{1}, x_{2}\right)$ of points within considered region. If "level surface" $y=$ const is outlined at the region surface, then the closed curves of level y that bound the spots are projected on it. Algorithms for simulation of spottiness are based on the numerical solution of the algebraic inequalities determining coordinates of internal points of spots. It is impossible to write the equation of spots contours in a general. Therefore contours of spots are described by system of the simple algebraic equations connected among themselves by equation $\Sigma \varphi_{i}(x, y)=0$, where $\varphi_{i}(x, y)$ is the equation of an elementary curve. For simplification of software realization of simulation of spottiness image as the equations $\varphi_{i}(x, y)$ the equation of a circle with varied coordinates of the centre and radius is accepted. Complex forms of spots are formed by overlapping on a plane of the drawing of several circles with different parameters that is defined by system of inequalities of a kind:

$$
\sum\left\{\left(x-a_{i}\right)^{2}+\left(y-b_{i}\right)^{2}-r_{i}\right\} \leq 0
$$

Where $x, y$ are the cartesian coordinates of internal points of spots, $a_{i}, b_{i}, r_{i}$ are coordinates of the centre and radius $i$-th circle, respectively, $n$ is quantity of the circles composing the modeled image. To simulate the randomness of background distribution for spottiness the spottiness model parameters $a_{i}, b_{i}$, $r_{i}$ are set by means of random-number generators. By changing laws of distribution of random numbers and their statistical parameters, it is possible to receive statistically different spottiness images.

Use the decimal system in Arabic figures for the numbering of headings and subheadings. Major (or section) headings are to be in capitals and bold.

\section{IDENTIFICATION THE SPOTS OF POLLUTANTS ON THE WATER SURFACE}

The problem of detection and identification of the pollution spots on the water surface, especially of oil spills, is being solved by many scientists. The recently developed technology of an adaptive identification of the environmental elements from measurements in the visible spectral region permits to synthesize an expert system for an adaptive identification of the environmental parameters (ESAIWP). The system's structure includes a compact multi-channel spectropolarimeter (MSP), information interface with computer (IIC), computer software (STW), and extending database (EDB). The STW realizes a number of algorithms to process the data fluxes from MSP and provides service functions of visualization and control of the regime of measurements. The EDB consists of the sets of standard spectral images of the spots of pollutants represented by points in the multi-dimensional vector space of indicators, pre-calculated on the basis of learning samples.

The principle of the ESAIWP functioning is based on fixation of changes of the light flux at the MSE output and their transformation into a digital code. Further processing of these data with respect to their efficiency is determined by the STW composition containing various algorithms of recognition of $2 \mathrm{D}$ objects. The adaptability of the recognition procedure is determined by the level of accumulated knowledge about special features of intensity fluctuations and polarizing properties of the light reflected from the water surface. The STW includes the means that make it possible, in case of uncertain identification of the pollution spot, to make an expert decision based on the visual analysis of its spectral image. This procedure is realized in the mode of dialogue with ESAIWP, and if decision is made, the operator can fix it in the database in the form of a standard for subsequent situations of an appearance of similar spots.

The principal scheme of the STW unit providing the procedure of identification is some transformation $\Phi$. The light intensity $\xi$ ${ }_{j}^{i}$ recorded at the time moment $t_{i}$ in the channel $\lambda_{j}$ is evaluated by the algorithm $\Phi$ where two hypotheses $H_{0}$ and $H_{1}$ are identified. The ESAIEP operator determines initial data $\mathbf{v}_{\boldsymbol{i}}, \alpha$ and $\beta$ and decides of which parameters $\mathbf{u}_{i}=\left(\mathrm{u}_{1}, \ldots, \mathrm{u}_{r}\right)$ will be calculated from measurements of $\left\{\xi^{j}{ }_{i}\right\}$. The service unit IIC makes it possible to form vector $\mathbf{u}_{i}$ from statistical characteristics of the series $\left\{\xi_{i}^{j}\right\}$ or to use direct measurements. A-priori information characterizes the type of distribution $f_{a}(\mathbf{u}$ i). The function

$$
L_{i}=\sum_{\hat{\imath}=1}^{\varnothing} \psi_{j}=f_{a 1}\left(\mathbf{u}_{i}^{j_{i}}\right) / f_{a 0}\left(\mathbf{u}_{\boldsymbol{i}}^{\boldsymbol{j}_{i}}\right)
$$

is compared with its ultimate values $L_{i, \min }$ and $L_{i, \max }$ At the first stage, these values are chosen arbitrarily, but then they change till reaching a maximum accurate recognition of the hypotheses $H_{0}$ and $H_{1}$. We have $L_{i, \min } \rightarrow L^{*}{ }_{i, \min }$ and $L_{i, \max } \rightarrow$ $L_{i, \max }^{*}$. The values $L^{*}{ }_{i, \min }$ and $L_{i, \max }^{*}$ are memorized in EDB. After the learning procedure, the functioning of the expert system is limited only by the volume of measurements fixed by the operator, proceeding from statistical reliability and the realtime regime. The operator has two possibilities to regulate this regime, establishing the volume of the series $\left\{\xi^{j}{ }_{i}\right\}$ or fixing the time of their accumulation. Usually the latter characteristic equal to $1 s$ is chosen. The operator is combined with the ESAIWP units through the man-machine interface IIC, which provides the selective control of operations in all units.

In the presence of the oil film on the water surface the system analyzes its thickness, age, source and geometry. In this case 
most informative are series of measurements at wavelengths $398 \mathrm{~nm}, 439 \mathrm{~nm}$ and $480 \mathrm{~nm}$. In the case of dissolved or suspended components in the water the system estimates their concentration and from the data in EDB on the hydrodynamic parameters of the water body, it calculates their spatial distribution using the methods of $2 \mathrm{D}$ images processing.

\section{REFERENCES}

F.A. Mkrtchyan, V.F. Krapivin, V.I. Kovalev, V.V. Klimov. An Adaptive Spectroellipsometer for Ecological Monitoring. Microwave and Optical Technology Letters. Volume 51, Issue 11, 2009, pp.2792-2795.

Ferdenant A. Mkrtchyan, Vladimir F. Krapivin, Vitaly I.

Kovalev and Vladimir V. Klimov. An Adaptive

Spectroellipsometric Technology for Ecological Monitoring of Sea Water. PICES Scientific Report, No.36, 2009, pp.215-218. 